\title{
Analytical design of the fractional order controller and robustness verification
}

\author{
Sateesh K. Vavilala, Vinopraba Thirumavalavan \\ Department of Electrical and Electronics Engineering, NIT Puducherry, Karaikal, India
}

\begin{tabular}{l} 
Article Info \\
\hline Article history: \\
Received Sep 13, 2020 \\
Revised Dec 1, 2020 \\
Accepted Feb 12, 2021 \\
\hline
\end{tabular}

\section{Keywords:}

Bode's ideal transfer function

Coupled tank system

Direct synthesis

Fractional order controller

Robust stability

\begin{abstract}
This work designs a fractional order controller (FOC) for the level control problem of the coupled tank system, using the desired time domain specifications. The direct synthesis method is employed to design the FOC. In the direct synthesis method, the Bode's ideal loop transfer function is chosen as the reference closed loop transfer function (CLTF). Bode's CLTF has the advantages like robustness to system gain variations, constant phase and very high gain margin. Performance of the proposed controller is compared with the state of the art literature. The simulation results showed better time domain performance of the proposed controller. The robust performance of the proposed controller is also the best. Robust stability of the system with the proposed controller is verified, and the system is found to be robustly stable.
\end{abstract}

This is an open access article under the $\underline{C C B Y-S A}$ license.

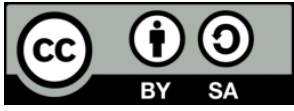

\section{Corresponding Author:}

Sateesh K. Vavilala

Department of Electrical and Electronics Engineering

National Institute of Technology Puducherry

Karaikal- 609609, India

Email: vksateesh@gmail.com

\section{INTRODUCTION}

The coupled tank system (CTS) is used in the process control industry for storing the liquid and pumping the liquid to next stages of the batch process. The interacting mode comprises two cylindrical shaped tanks connected physically through a valve. The level control problem of the CTS is an interesting problem due to its nonlinear nature and delay involved. John et al., proposed a backstepping controller for the CTS [1]. Sharma et al., discussed the modeling of the CTS and proposed a fuzzy logic controller for the height control problem [2]. Sathish Kumar et al., developed a nonlinear model of the three tank system and designed a linear quadratic controller [3]. Patel and Shah designed an integer order proportional integral differentiator (PID) and artificial intelligence based controllers for the height control of the CTS [4]. Singla et al., proposed a modified PID controller for the level control of the CTS [5]. The modified PID controller showed better performance than the conventional PID controller. Gouta et al., discussed the adaptive control of coupled tank system using an observer [6]. A multistage cost function is optimized to obtain the controller parameters. Mahapatro et al., designed a decentralized proportional integral (PI) controller for the height control of a CTS using a reference model [7]. A frequency domain based modeling of the system is also discussed. Nafea et al., discussed a neural network based height control of the CTS [8]. They proposed a perturbed model of the system from the nonlinear model. Apart from the conventional controllers; fractional order controllers (FOC) are employed for the level control of the tanks.

FOCs are finding applications in all areas of control systems because of advantages like isodamping property, choice of controller parameters. Podlubny proposed the first fractional order proportional integral derivative (FOPID) controller [9]. After FOPID is developed, numerous other fractional controllers are 
designed and implemented. Vinopraba et al., discussed two degrees of freedom (2-DOF) FOC [10]. The parameters are tuned analytically using the desired closed loop bandwidth. Saxena and Hote discussed an internal model controller-proportional integral differentiator (IMC-PID) based fractional controller for the DC servomotor speed control [11]. The controller has less tunable parameters and the controller parameters are obtained using frequency domain specifications. Baruah et al., proposed a fractional order proportional integral (FOPI) controller for the CTS. The parameters of the controller are obtained analytically using integral performance index and balanced state space methods [12]. Walid et al., developed a FOPID controller for the speed control of permanent magnet synchronous machine [13]. The controller is synthesized using the Bode's ideal open loop transfer function (OLTF). Lino et al., proposed a feed forward fractional controller for the speed and position control of DC motor [14]. Empirical formulae for the controller parameters are obtained using the frequency domain specifications. Zhenlog et al., devised a Smith predictor based FOPI controller for the higher order system [15]. The controller gave good servo and disturbance responses. Vavilala and Vinopraba tuned the FOC parameters using particle swarm optimization (PSO) algorithm for a conical tank system level control [16]. A FO- $[P I]^{\lambda}$ controller is developed for the two input two output (TITO) CTS using the frequency domain specifications [17]. Fractional powers are approximated using Oustaloup recursive approximation (ORA). Teplijakov proposed a toolbox called fractional order modeling and control (FOMCON) in MATLAB for implementing fractional controllers and modeling the fractional order systems [18]. The FOMCON toolbox is used for simulations in this work.

The FOC parameters can be tuned using analytical procedure, evolutionary algorithms and empirical formulae. In this work, controller structure is synthesized analytically using the direct synthesis procedure, which is a conventional analytical method used for the controller design. A desired model of the system will be specified and using that model, controller will be synthesized. Vanavil et al., discussed the design of PID controller using the direct synthesis for the time delay systems [19]. The parameters of the controller got using the maximum. Ravikishore et al., used the direct synthesis method to derive the PID controller for the unstable first order and seconder order time delay systems [20]. Castillo-Garcia et al., proposed a FO- [PI] ${ }^{\alpha}$ controller for the automation of the water canals [21]. Kumar and Anwar discussed a practical application using the direct synthesis [22]. The controller is designed using the maximum sensitivity. Direct synthesis based controller design is discussed in [23-25]. Anil and Padmasree used direct synthesis method to obtain the PID controller for different integrating systems with time delay [23]. Anwar and Pan utilized the direct synthesis method to get the PID controller parameters for the load frequency control (LFC) problem [24]. Kumar and Singh designed a PID controller for six different time delay systems using the direct synthesis method [25]. This work considers Bode's ideal OLTF as the desired reference model for the closed loop system. Bode [26] proposed the OLTF as a reference model.

Kasbi and Rahali proposed a FOPI controller for the wind generation system using the Bode's ideal OLTF [27]. Ye and Bing discussed the design of the Smith predictor based 2-DOF IMC using the Bode's ideal OLTF method as the desired model [28]. The controller parameters are optimized using the integral absolute error. Yumuk et al., proposed a FOPID based on the Bode's ideal OLTF for the fractional order system [29]. Frequency domain specifications are used to tune the controller parameters. In a recent work, Keziz et al., discussed the design of FOC with Bode's ideal OLTF as a reference model and pole placement technique for controller tuning [30]. The controller is simple and robust. Safaei and Tavakoli proposed an analytical procedure for the FOC design using direct synthesis [31]. The controller parameters are tuned using the time domain specifications with empirical formulae. Arya and Chakrabarty designed a FOIMC for the nonminimum phase systems [32]. The controller parameters are optimized using the time domain specifications and integral performance indices. Paliwal et al., discussed the design of PID controller for the LFC problem [33]. PSO algorithm is used to get the controller parameters using different performance indices. A robust controller handles the uncertainty in the system parameters, rejects the disturbances, and performs well for changes in the reference input. The designed controller has to perform reasonably well for a change in the operating point. Robust performance and robust stability are assessed with $H_{\text {回 }}$ norm [34]. In a recent work by the authors direct synthesis is employed for obtaining the controller [35].

Purpose or motivation of the work: Most of the literature on the controller design, based on the Bode's ideal closed loop transfer function (CLTF), tuned the controller parameters using the frequency domain specifications. But [31] proposed a Smith predictor based FOC using the time domain specifications. The empirical formulae are devised based on the Bode's ideal CLTF step response. These formulae can be used for any linear system with a time delay. The controller is designed using the direct synthesis approach. Motivated by the simple design procedure based on the time domain specifications, an attempt is made to design a FOC without the Smith predictor, for the first order plus delay time (FOPDT) model of the system. The proposed controller handled the time delay in the system without the Smith predictor. For the systems with large time delay and/or large time constants, the controller parameter tuning using the frequency domain specifications will not give satisfactory results, hence time domain specifications based tuning is more apt. 
Main contributions and novelty: A novel FOC configuration based on the Bode's ideal OLTF is obtained is proposed. Servo response, disturbance responses are compared with the state of the art. Robust performance and robust stability of the proposed FOC are analyzed using $H_{\square}$ norm. The fragility indices of the controllers are compared to check the fragility of the controller to changes in the controller parameters. The proposed controller has the simple structure of a PI controller cascaded with a fractional order filter. The controller parameters are tuned using the desired peak overshoot (PO) and settling time $\left(t_{s}\right)$ from the desired step response. Novelty in the controller design is; FOC is analytically synthesized without the Smith predictor, for a FOPDT model of the system.

Organization of the paper: The second section describes the problem formulation and preliminaries of the fractional order control. In the third section analytical design of the proposed FOC is discussed. Robust stability, robust performance and fragility analysis methods are discussed in the third section. The fourth section discusses the simulation results. The fifth section concludes the paper.

\section{PROBLEM FORMULATION AND PRELIMINARIES}

\subsection{Problem formulation}

Statement of the problem and the proposed solution: This work is aimed at addressing the height control of CTS based on the time domain specifications like PO and $t_{s}$. The work provides the solution for this problem by proposing a design based on the empirical formulae involving the peak overshoot and settling time. Modified Bode's ideal CLTF is taken as the desired transfer function and using the direct synthesis method, controller is derived. The work also verifies whether the performance of the proposed FOC is robust enough and robustly stable. The study also discusses the existing literature on the FOC design using time domain specifications. For the FOPDT model of the CTS, a FOC is designed such that the following controller objectives are satisfied.

Controller objectives:

- $\quad$ Achieve good transient and steady state step responses $\left(M_{p}<=10 \%\right.$ and $\left.t_{s}<=150 \mathrm{sec}\right)$.

- $\quad$ Achieve a satisfactory set point tracking and reject the disturbance simultaneously.

- Obtain a stable and robust controller.

Figure 1 shows the block diagram of the feedback controller. In Figure 1, the system $G(s)$ has the FOPDT model given by (1), and the FOC $C(s)$ design is discussed in Section 3.



Figure 1. Feedback controller

$$
\mathrm{G}(\mathrm{s})=\frac{K e^{-L s}}{T s+1}
$$

\subsection{Preliminaries of fractional calculus and fractional control}

Fractional calculus is 300 years old. In the last thirty years fractional controllers are widely used. The Caputo fractional derivative is given by (2).

$$
\mathrm{D}_{t}^{\alpha} f(t)=\frac{1}{\Gamma(n-\alpha)} \int_{0}^{t} \frac{f^{n}(\tau)}{(t-\tau)^{\alpha-n+1}} d \tau
$$

FOPID controller developed by I. Podlubny is represented as (3).

$$
C(s)=K_{p}+\frac{K_{i}}{s^{\lambda}}+K_{d} s^{\mu}
$$

where $K_{p}, K_{i}$, and $K_{d}$ are proportional, integral and derivative gains respectively. After FOPID, many other fractional order controller configurations are proposed and are widely used. 


\section{RESEARCH METHOD}

\subsection{Design of the fractional order controller}

Bode's ideal OLTF is the reference for many design procedures in the fractional controllers. The (4) shows the Bode's OLTF denoted by $L(s)$.

$$
\begin{aligned}
& L(s)=\frac{1}{\tau_{c} s^{\alpha}} \\
& \tau_{c}=\frac{1}{\left(\omega_{g c}\right)^{\alpha}}
\end{aligned}
$$

where,

$\tau_{c}=$ Time constant

$\omega_{g c}=$ Gain crossover frequency

$\alpha=$ Fractional order $(1 \leq \alpha<2)$

(5) gives the relation between $\tau_{c}$ and $\omega_{g c}$. The CLTF $T(s)$ with $L(s)$ in forward path is,

$$
T(s)=\frac{1}{1+\tau_{c} s^{\alpha}}
$$

The (6) is used to get the step responses are obtained using the (6) for different values of $\alpha$ [31]. The following (7-11) represent the relation among PO $\left(M_{p}\right)$, fractional order $(\alpha)$, settling time $\left(t_{s}\right)$ and GCF $\left(\omega_{g c}\right)$ :

$$
\begin{aligned}
& M_{p}=73.9\left(\alpha^{2}-1.6739 \alpha+0.6756\right) \\
& t_{s-2 \%} \omega_{g c}=\frac{0.7885 \alpha-0.2693}{\alpha-0.8673}, 1<\alpha<1.078 \\
& t_{s-2 \%} \omega_{g c}=\frac{3.003 \alpha-2.981}{\alpha^{2}-2.012 \alpha+1.056}, 1.078<\alpha<1.486 \\
& t_{s-5 \%} \omega_{g c}=\frac{0.812 \alpha-0.2036}{\alpha-0.8007}, 1<\alpha<1.15 \\
& t_{s-5 \%} \omega_{g c}=\frac{7.156 \alpha-7.9}{\alpha^{2}-1.303 \alpha+0.2578}, 1.15<\alpha<1.5
\end{aligned}
$$

In this work (10) is implemented since it has PO less than 3\%. (6) is the modified with time delay incorporated and given by (12). Figure 2 shows the block diagram representation of the (12).

$$
T_{\text {new }}(s)=\left(\frac{Y(s)}{R(s)}\right)_{\text {desired }}=\frac{e^{-L s}}{1+\tau_{c} s^{\alpha}}
$$

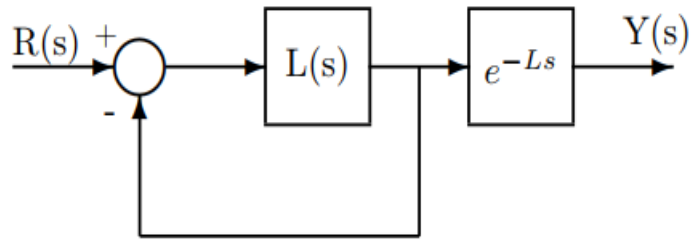

Figure 2. Desired closed loop reference transfer function

(13) shows the transfer function of any closed system with the controller $C(s)$ and system transfer function $G$ $(s)$.

$$
\frac{Y(S)}{R(S)}=\frac{G(s) C(s)}{1+G(s) C(s)}
$$

If the desired CLTF of (12) does not include the time delay element $e^{-L s}$, then the synthesized controller $C(s)$ will have an unstable pole at $1 / L$, hence including the time delay element in (12) is mandatory. Substituting $G(s)$ from (1) in (13), 


$$
\frac{Y(S)}{R(S)}=\frac{\left(\frac{K e^{-L s}}{T s+1}\right) C(s)}{1+\left(\frac{K e^{-L S}}{T s+1}\right) C(s)}
$$

Comparing (13) and (14) and solving for $C(s)$,

$$
C(s)=\left(\frac{T s+1}{K}\right)\left(\frac{1}{1+\tau_{c} s^{\alpha}-e^{-L s}}\right)
$$

Expanding the delay term $e^{-L s}$ in the denominator, using the Taylor series of first order and simplifying further,

$$
C(s)=\left(\frac{T s+1}{K}\right)\left(\frac{1}{\tau_{c^{s}}{ }^{\alpha}+L s}\right)
$$

Rearranging terms of (16),

$$
C(s)=K_{c}\left(1+\frac{1}{T_{i} s}\right)\left(\frac{1}{1+\left(\tau_{c} / L\right) s^{\alpha-1}}\right)
$$

the structure of $C(s)$ is a PI cascaded with a FO filter of order $(\alpha-1)$ where,

$K_{c}=T / L K=$ Proportional gain constant

$T_{i}=T=$ Integral time constant

From the chosen values of $M_{p}, t_{s}$ and using (7) and (10) $\alpha, \tau_{c}$ are obtained. The design procedure is explained in the following steps:

- $\quad$ Obtain FOPDT model of the system.

- $\quad$ Decide on the required time domain specifications, PO and $t_{s}$.

- $\quad$ Using the empirical formulae, get the controller parameters of FOC.

- $\quad$ Using the MATLAB/ SIMULINK simulation, obtain the step responses and verify if the time domain specifications are met?

Advantages of the proposed FOC:

- Isodamping property.

- $\quad$ Infinite gain margin.

- Increased stability range.

\subsection{Robust stability and robust performance analysis}

For a change in the set point, the controller should give reasonable performance. The (18) shows the criteria to be satisfied for the controller to be robust [34].

$$
20 \log _{10}\left(\mid T_{c}(j \omega) \| G_{\Delta}(j \omega \mid)<0 d B\right.
$$

where $T_{c}(s)$ is the complementary sensitivity.

$$
\tau_{c}(s)=\frac{G(s) C(s)}{1+G(s) C(s)}
$$

expanding te system transfer function $G(s)$ using Taylor series of first order,

$$
G(s)=\frac{-K L s+K}{T s+1}
$$

substituting (16) and (20) in (19), and simplifying,

$$
\tau_{c}(s)=\frac{1-L s}{1+\tau_{c} s^{\alpha}}
$$

the (22) shows the new transfer function at the new set point.

$$
G_{1}(s)=\frac{K_{1} e-^{L_{1} s}}{T_{1} s+1}=\frac{-K_{1} L_{1} s+K_{1}}{T_{1} s+1}
$$


uncertainty $G_{\Delta}(s)$ is obtained as,

$$
G_{\Delta}(s)=\frac{G_{1}(s)-G(s)}{G(s)}
$$

substituting (20) and (22) in (23), $G_{\Delta}(s)$ is obtained as shown in (24).

$$
G_{\Delta}(s)=\frac{\left(K L T_{1}-K_{1} L_{1} T\right) s^{2}+\left(K\left(L-T_{1}\right)-K_{1}\left(L_{1}-T\right)\right) s+K_{1}}{K L T s^{2}+\left(K T_{1}-K L\right) s+K}
$$

the $(25)$ is to be satisfied by the controller for getting robust performance.

$$
20 \log _{10}\left(\left|T_{c}(j \omega) \| G_{\Delta}(j \omega)\right|+|S(J \omega)||W(j \omega)|<<0 d B\right.
$$

the sensitivity transfer function $S(s)$ is given by,

$$
S(s)=\frac{Y(s)}{d(s)}=\frac{1}{1+G(s) C(s)}
$$

substituting (16) and (20) in (26),

$$
S(s)=\frac{L s+\tau_{c^{s}} a}{1+\tau_{c^{S}}{ }^{a}}
$$

$W(s)$ is a low frequency weighting function, since $S(s)$ needs to be minimized only in the low frequency disturbances. In the present work, the weight is selected as,

$$
W(s)=\frac{0.01 s+0.001}{s}
$$

The Bode plot of the weight is shown in Figure 3. It is selected to have a high magnitude in lower frequencies, and low magnitude in higher frequencies

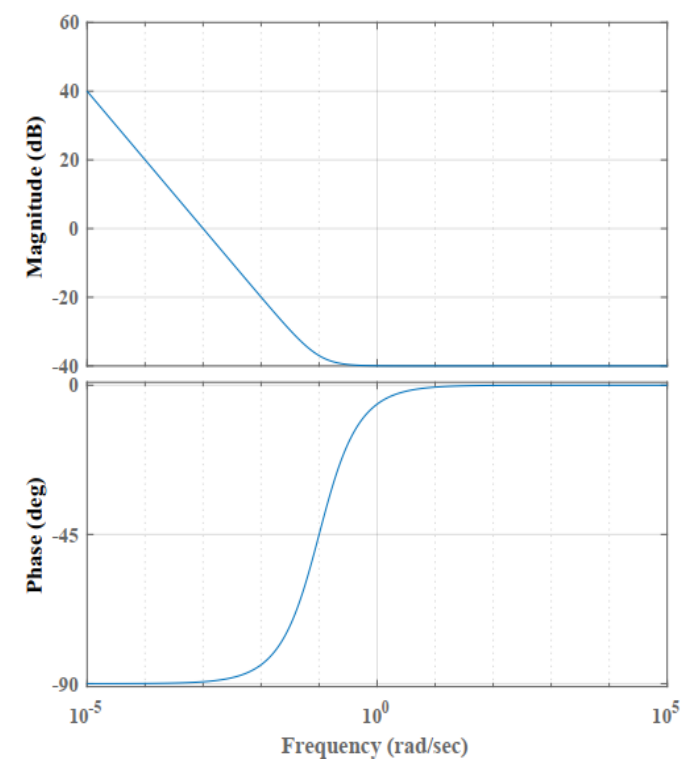

Figure 3. Bode plot of weight function $W(s)$

\subsection{Fragility analysis}

Apart from robustness to system parameter variation, robustness to controller parameter variation needs attention. While analog controller implementation suffers from physical parameter changes [32], digital controller implementation suffers from the inaccuracies in fixed word length and roundoffs in 
numerical calculation. The robustness of the controller parameter variation is analyzed using fragility index $R F I_{\Delta 20}$ [32] defined by (29).

$$
R F I_{\Delta 20}=\left|\frac{M_{S \Delta 20}}{M_{S}}-1\right|
$$

where $M_{s}$ is the nominal maximum sensitivity and $M_{s 420}$ is the nominal maximum sensitivity obtained when all the controller parameters are varied by $+20 \%$. The controller is said to be resilient to controller parameter changes, for values of the $R F I_{\Delta 20} \leq 0.1$, is nonfragile for values of $0.1<R F I \Delta 20 \leq 0.5$ and fragile for values of $R F I_{\Delta 20}>0.5$.

\section{RESULTS AND DISCUSSION}

\subsection{Case study: Coupled tank system}

In this work CTS case study is considered to verify the effectiveness of the proposed FOC. Figure 4 shows the block diagram of the CTS and, Table 1 give the nominal parameters of the CTS.

$Q_{l}=$ Flow rate to tank one

$Q_{12}=$ Flow rate between the tanks

$Q_{d l}=$ Disturbance flow rate of first tank

$Q_{d 2}=$ Disturbance flow rate of second tank

$h_{l}=$ Level of the tank one

$h_{2}=$ Level of tank two

$D=$ Diameter tank

$A=$ Cross sectional area of tank

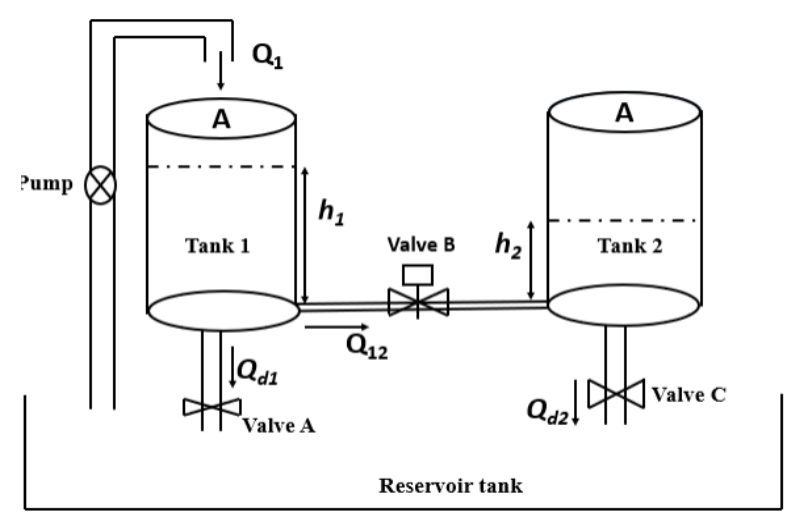

Figure 4. Cylindrical coupled tank system control

By varying the flow rate to first tank second tank level has to be controlled. The operating region selected is $(5-15) \mathrm{cm}$. Figure 5 shows the open loop test results got from the two tank system by changing the flow rate $Q_{1}$ from (50-225) LPH. The open loop input and output data shows that the coupled tank system level control in the operating height of $(5-15) \mathrm{cm}$ follows a first order FOPDT model (in the (5-15) $\mathrm{cm}$ range) dynamics. From the experimental open loop data, FOPDT model of the system in the operating height of (515) $\mathrm{cm}$ is obtained as,

$$
G(s)=\frac{h_{2}(s)}{Q_{1}(s)}=\frac{0.268 e^{-10 s}}{975 s+1}
$$

Table 1. Nominal tank parameters

\begin{tabular}{cc}
\hline Parameter & Value \\
\hline$H$ & $100 \mathrm{~cm}$ \\
$D$ & $15 \mathrm{~cm}$ \\
$A$ & $176.7 \mathrm{~cm}^{2}$ \\
$Q_{I}$ & $175 \mathrm{LPH}$ \\
\hline
\end{tabular}




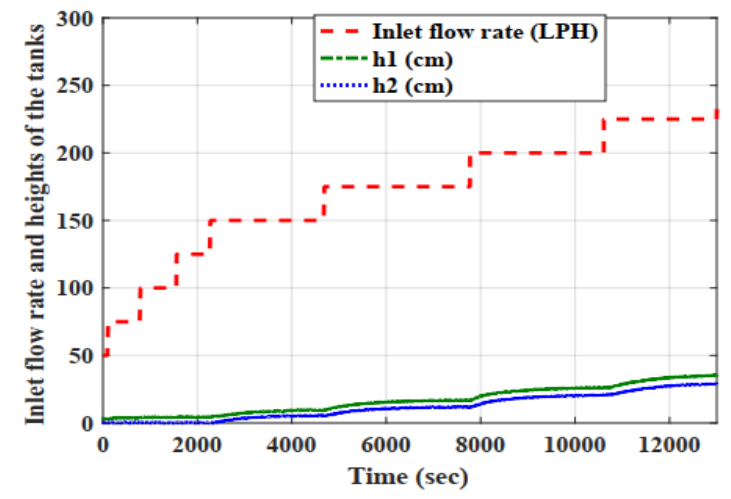

Figure 5. Open loop test results of level

\subsection{Time domain results}

The time domain specifications selected for the controller design are, $M_{p}<=10 \%$ and $t_{s}<=150 \mathrm{sec}$. In (7) by substituting $M_{p}$ with 0.1 and solving for the $\alpha$, fractional order is obtained 1.15. In (10), substituting $t_{s}$ with 150 and $\alpha$ with 1.15 and $\omega_{g c}$ is obtained as $0.0208 \mathrm{rad} / \mathrm{sec}$. Finally using (5) and substituting the values of $\omega_{g c}$ and $\alpha, \tau_{c}$ is obtained as 85.56. The fractional controller derived for the CTS is shown in (31).

$$
C_{F O C}(s)=\left(363.8+\frac{0.373}{s}\right)\left(\frac{1}{1+8.55 s^{0.15}}\right)
$$

using (21) and (27), $T_{C}(\mathrm{~s})$ and $S(s)$ are obtained as,

$$
\begin{aligned}
& T_{c}(s)=\frac{1-10 s}{1+85.65 s^{1.15}} \\
& S(s)=\frac{10 s+293 s^{1.15}}{1+293 s^{1.15}}
\end{aligned}
$$

Sometimes the controller has to accommodate the changes (be robust) in the plant parameters, changes in the setpoint, changes in the operating region. The transfer function of the new operating region $(16-30) \mathrm{cm}$ is (34).

$$
G_{1}(s)=\frac{0.3 e^{-20 s}}{1095 s+1}
$$

using (23) $G_{\Delta}(s)$ is obtained as,

$$
G_{\Delta}(s)=\frac{-2915.4 s^{2}-4.28 s+0.032}{-2934 s^{2}+290.78 s+0.268}
$$

A FOIMC designed for the CTS is denoted as Ref-1 [32]. The controller obtained is,

$$
C_{\text {FOIMC-Ref-1 }}(s)=\frac{975 s+1}{3.85 s^{1.23}+2.68 s}
$$

integer order internal model controller (IOIMC) for the CTS is taken as Ref-2 and is obtained as,

$$
C_{\text {FOIMC-Ref-2 }}(s)=12.16+\frac{0.012}{s}
$$

To compare the performance of the FOMCON toolbox, the standard CRONE toolbox and controller implementation is considered. To handle the time delay in the system, the second-generation CRONE controller along with Smith predictor is used. The frequency domain specifications used for the CRONE controller design are GCF of $0.08 \mathrm{rad} / \mathrm{sec}$ and PM of 45 degrees. The FOC obtained using CRONE is given by (38).

$$
C_{C R O N E}(s)=\frac{661.2 s+0.678}{s^{1.479}}
$$


For getting the simulation results, Figure 1 is implemented in the MATLAB/SIMULINK with the $C$ $(s)$ given by (31) and $G(s)$ given by (30). The set point $R$ for the tank 2 height, is selected as $10 \mathrm{~cm}$. FOMCON toolbox provides SIMULINK blocks for fractional transfer function, FOPID controller transfer functions. Using these blocks, the step responses of the system for a set point of $10 \mathrm{~cm}$ are obtained with different controllers and are shown in Figure 6. Upper portion of Figure 6 shows the step responses, whereas bottom portion shows the corresponding control efforts of each controller in a scale of (0-100)\%. Figure 6 , shows that the proposed FOC has good transient and steady state performance. Novelty of these results is that, they are obtained from the time domain specifications directly. Figure 7 gives the regulatory responses. Figure 7 shows that the disturbance applied at $500 \mathrm{sec}$ settles at $600 \mathrm{sec}$. While the controller, FOIMC settled at $610 \mathrm{sec}$ and IOIMC settled at $620 \mathrm{sec}$. Hence the proposed controller has good disturbance rejection.


Figure 6. Step responses and control efforts

Figure 7. Regulatory responses and control efforts

Figure 8 gives the servo tracking responses. Figure 8 shows that the proposed controller gave good step response for changes in the step input. The Table 2 shows the time domain specifications and time domain indices. Control effort indicates the power consumed in obtaining the desired control action. The CRONE controller has the lowest integral absolute value. The CRONE controller toolbox consumes the highest control energy. The peak overshoot of the CRONE toolbox is also the highest among all the controllers. But the CRONE controller has the least $t_{\mathrm{s}}$ and the least rise time.


Figure 8. Servo responses and control efforts

IAES Int J Rob \& Autom, Vol. 10, No. 1, March 2021 :`10 - 23 
The Table 2, shows the controller satisfies the controller objectives, $M_{p}<=10 \%$ and $t_{s}<=150 \mathrm{sec}$, which proves the validity of the design procedure. Hardships faced during the design of the proposed FOC are difficulty in choosing proper weighting function $W(s)$, proper time domain specifications. The time domain specifications must not be too much ambitious and the weighting function should have proper bandwidth. The proposed controller has the least time domain specifications. Figure 9 shows the robustness of the controllers to set point change from $10 \mathrm{~cm}$ to $20 \mathrm{~cm}$. The proposed controller showed an increase of $10 \%$ to $4 \%$ in peak overshoot. For the FOIMC, peak overshoot has increased by from $25 \%$ to $75 \%$. The rise time reduced from $900 \mathrm{sec}$ to $500 \mathrm{sec}$ and settling time reduced from $3600 \mathrm{sec}$ to $2600 \mathrm{sec}$. From this discussion, the proposed controller gave superior robustness. The system gain is varied by $\pm 20 \%$ to verify the robustness of the controller. Figure 10 and Figure 11, show that the controller is robust to parameter changes.

Table 2. Time domain performance specifications of the controllers

\begin{tabular}{ccccc}
\hline Parameter & & \multicolumn{2}{c}{ Controller } & \\
& FOC (Proposed) & FOIMC & IOIMC & CRONE \\
\hline IAE & 628 & 269.3 & 3034 & 62.36 \\
ISE & $3.562 \times 10^{3}$ & $1.7 \times 10^{4}$ & $1.612 \times 10^{4}$ & 273.5 \\
ITAE & $5.9 \times 10^{4}$ & $9.7 \times 10^{3}$ & $8.547 \times 10^{5}$ & 1300 \\
ITSE & $4.9 \times 10^{6}$ & $2.45 \times 10^{6}$ & $2.257 \times 10^{7}$ & $5.469 \times 10^{5}$ \\
$t_{r}(\mathrm{sec})$ & 35 & 25 & 150 & 15 \\
$t_{s}(\mathrm{sec})$ & 400 & 100 & 1200 & 35 \\
$M_{p}(\%)$ & 10 & 25 & 0 & 27 \\
$e_{s s}$ & 0 & 0 & 0 & 0 \\
$\int u^{2}$ & $1.759 \times 10^{7}$ & $7.868 \times 10^{7}$ & $5.026 \times 10^{6}$ & $4.23 \times 10^{8}$ \\
\hline
\end{tabular}
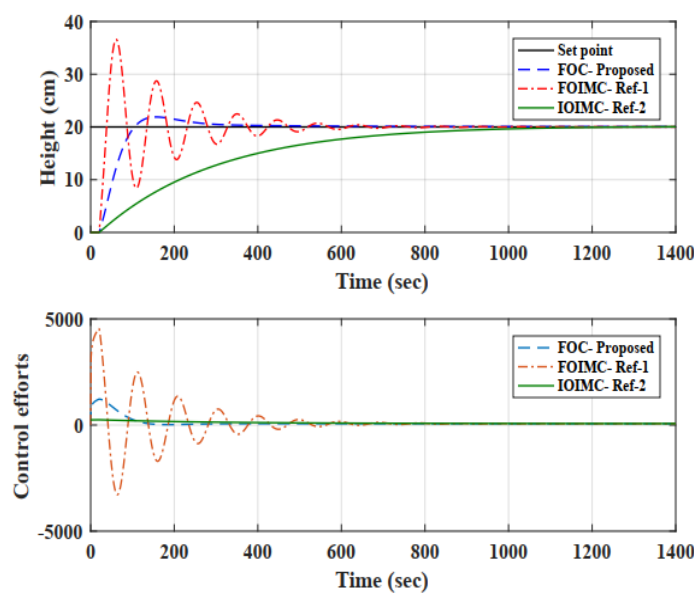

Figure 9. Verification of robustness
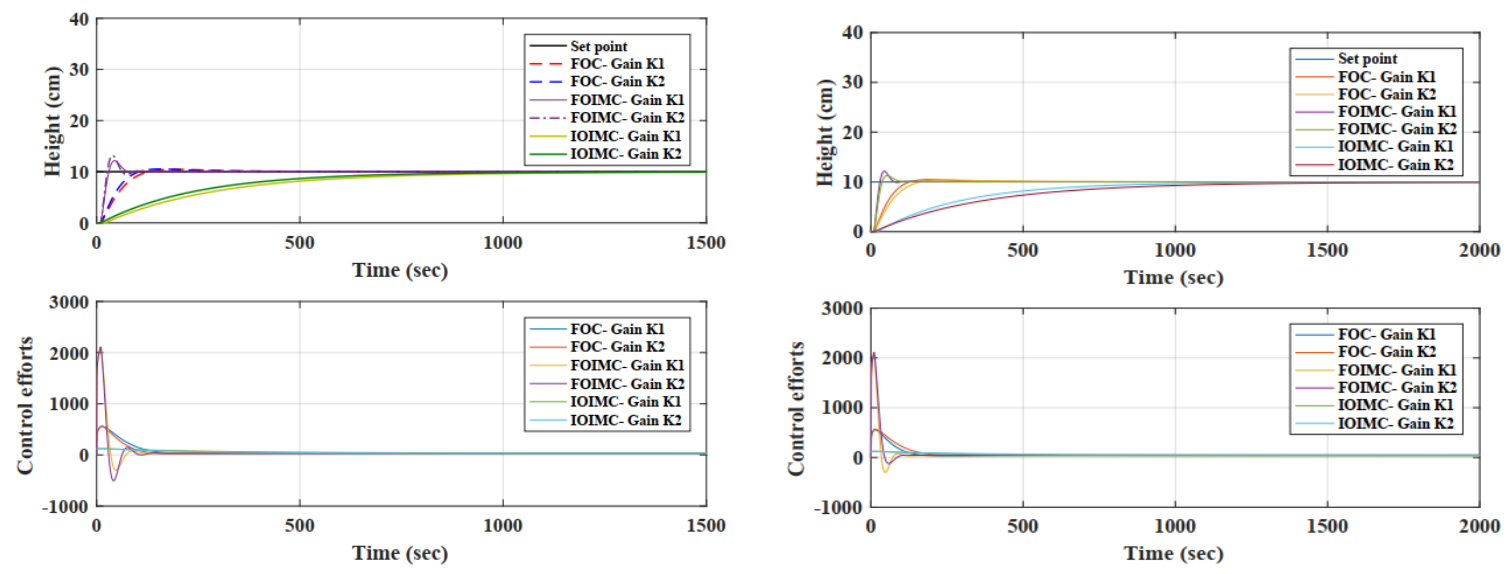

Figure 10. Step responses to gain change of $+20 \%$

Figure 11. Step responses to gain change of $-20 \%$ 
Table 3 compares the robust stability norms and fragility indices of the controllers. The proposed controller and Ref- 2 are resilient, Ref- 1 is nonfragile when all of the controller parameters are varied by $+20 \%$. The proposed controller and Ref- 2 have robust stability norms less than $0 \mathrm{~dB}$; hence the proposed controller and Ref- 2 are robustly stable. The Ref- 1 has robust stability norm of $0.59 \mathrm{~dB}(>0 \mathrm{~dB})$; hence Ref-1 is not robustly stable. The proposed controller and Ref- 2 have robust performance norms less than $0 \mathrm{~dB}$; hence the proposed controller and Ref- 2 will give good robust performance. The Ref- 1 has robust stability norm of $0.408 \mathrm{~dB}(>0 \mathrm{~dB})$, hence Ref-1 will not give good robust performance.

Table 3. Stability and robustness analysis

\begin{tabular}{cccc}
\hline \multirow{2}{*}{ Parameter } & \multicolumn{3}{c}{ Controller } \\
\cline { 2 - 4 } & FOC (Proposed) & FOIMC & IOIMC \\
\hline$R F I_{\Delta 20}$ & 0.087 & 0.345 & 0.00207 \\
Stability & -4.05 & 0.59 & -0.056 \\
norm $(\mathrm{dB})$ & & & -0.0566 \\
$\begin{array}{c}\text { Performance } \\
\text { norm }(\mathrm{dB})\end{array}$ & -4.3 & 0.408 & \\
\hline
\end{tabular}

\subsection{Frequency domain analysis}

Figure 12 shows the Bode plots of the uncontrolled system and controlled system. OLTF of the system has a flat phase response around the $G C F$ of $0.208 \mathrm{rad} / \mathrm{sec}$. Figure 13 compares the Bode plots of the controllers. Figure 13 shows that the proposed controller has good isodamping property and low pass frequency response. Figure 14 compares Bode plots of OLTF with the different controllers at a new set point of $20 \mathrm{~cm}$. Figure 14 indicates that at a different operating point also the proposed controller isodamping property.

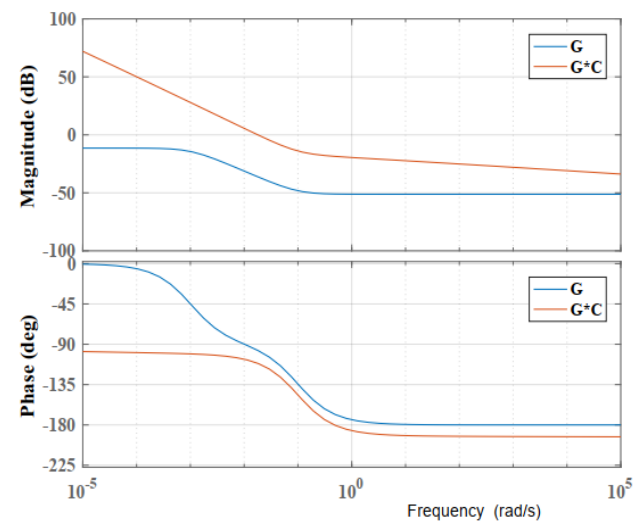

Figure 12. Bode plots of controlled and uncontrolled system

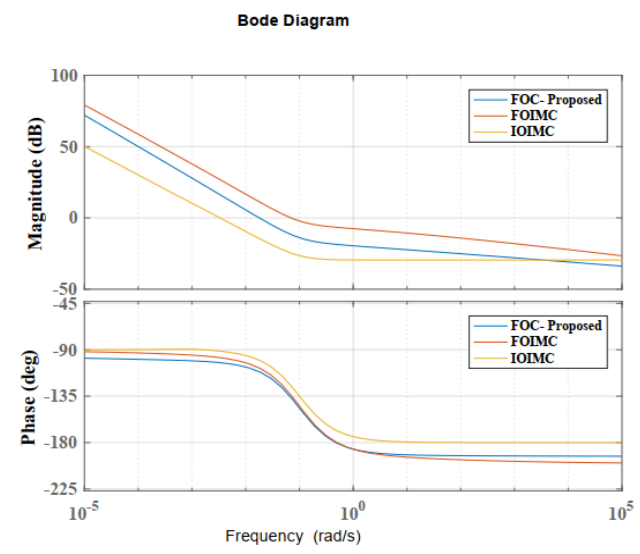

Figure 13. Bode plots of the controllers 
Peak overshoot of the proposed controller is lesser by $25 \%$ compared to FOIMC and lesser by $10 \%$ compared to IOIMC. The settling time of the proposed controller is $200 \%$ lesser than the FOIMC, and $285 \%$ lesser than IOIMC. The proposed controller satisfied the robust stability and robust performance infinity norms. Frequency domain analysis of the proposed controller and other controllers is done. Bode plots of the loop transfer function showed that the proposed controller has better isodamping property. Bode plots of the controller at two set points established the robustness property of the proposed controller. From the results it is concluded that, the novel configuration of the FOIMC tuning based on the time domain specifications achieves the controller objectives. The transfer function of the system considered is a generalized FOPDT model, hence the results obtained with the proposed novel controller are applicable for any other FOPDT system other than the coupled tank system.

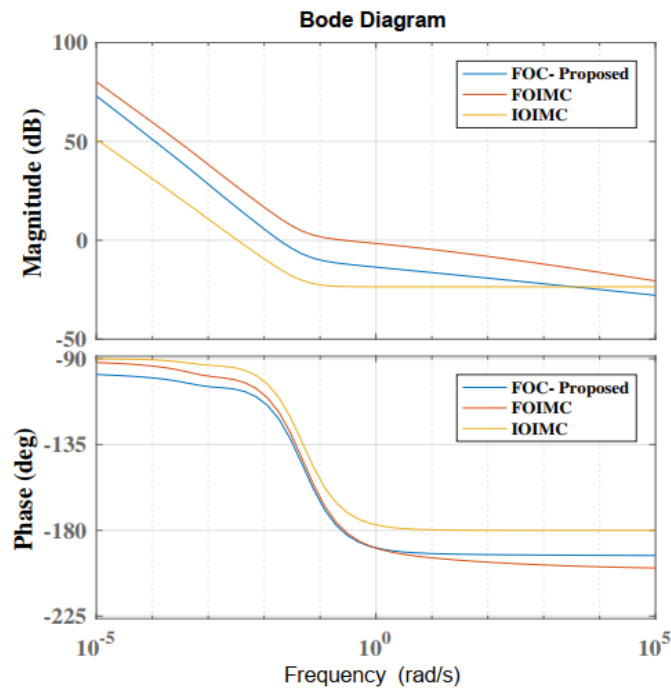

Figure 14. Comparison of bode plots at new operating point

\section{CONCLUSION}

This work designs a FOC for the level control of a CTS. A FOPDT model of the CTS is considered. The FOC is designed analytically based on the specified PO and $t_{s}$. Modified Bode's ideal CLTF is taken as reference and the controller is synthesized directly. Performance of the proposed controller is compared with state of the art. The proposed controller satisfied the time domain specifications used for the design. The FOC implementation using the FOMCON toolbox is compared with the CRONE toolbox. The CRONE toolbox based FOC consumed more control energy for achieving the control action when compared to the FOMCON toolbox based implementation.

\section{REFERENCES}

[1] J. A. John, N. E. Jaffar, R. M. Francis, "Modelling and control of coupled tank liquid level system using backstepping method," International Journal of Engineering Research \& Technology, vol. 4, no. 6, pp. 667-671, 2015.

[2] A. Sharma, O. P. Verma, M. Singh, "Mathematical modeling and intelligent control of two coupled tank system," Imperial Journal of Interdisciplinary Research, vol. 2, no. 10, pp. 1589-1593, 2016.

[3] K. Sathish Kumar, V. Kirubakaran, T. K. Radhakrishnan, "Real time modeling and control of three tank hybrid system," Chemical Product and Process Modelling, vol. 13, no. 1, pp. 1-10, 2012, doi: 10.1515/cppm-2017-0016.

[4] H. R. Patel , V. A. Shah, "A framework for fault tolerant control for an interacting and non-interacting level control system using AI," in Proceedings of the 15th International Conference on Informatics in Control, Automation and Robotics ICINCO, vol. 1, pp. 180-190, 2018, doi: 10.5220/0006862001800190.

[5] R. Singla, A. Agrawal, V. Kumar, N. Pachauri, O. P. Verma, "Industrial simulation of PID and modified M-PID controller for coupled tank system," in Advances in Signal Processing and Communications, Springer, Lecture Notes in Electrical Engineering, vol. 526, pp. 421-428, 2019, doi: 10.1007/978-981-13-2553-3_41.

[6] H. Gouta, S. H. Saïd, A. Turki, F. M'Sahli, "Experimental sensor less control for a coupled tank system using high gain adaptive observer and nonlinear generalized predictive strategy," ISA Transactions, vol. 87, pp. 187-199, 2019, doi: 10.1016/j.isatra.2018.11.046. 
[7] S. R. Mahapatro, B. Subudhi, S. Ghosh, "Design and experimental realization of robust decentralized PI controller for a coupled tank system," ISA Transactions, vol. 89, pp. 158-168, 2019, doi: 10.1016/j.isatra.2018.12.003.

[8] M. Nafea, A. R. M. Ali, J. Baliah, M. Sultan, M. Ali, "Metamodel-based optimization of a PID controller parameters for a coupled-tank system," Telecommunication, Computing, Electronics and Control, vol. 16, no. 4, pp. 1590-1596, 2018, doi: 10.12928/TELKOMNIKA.v16i4.9069.

[9] I. Podlubny, "Fractional order systems and fractional controllers," Slovak Academy of Sciences, Institute of experimental Physics, pp. 1-17, 1994.

[10] T. Vinopraba, N. Sivakumaran, S. Narayanan, T. K. Radhakrishnan, "Design of internal model control based fractional order PID controller," Journal of Control Theory Applications, vol. 10, no. 3, pp. 297-302, 2012, doi: 10.1007/s11768-012-1044-4.

[11] S. Saxena, et al, "Design and validation of fractional-order control scheme for DC servomotor via internal model control approach," IETE Technical Review, vol. 36, no. 1, pp. 49-60, 2017, doi: 10.1080/02564602.2017.1396935.

[12] G. Baruah, S. Majhi, C. Mahanta, "Design of FOPI controller for time delay systems and its experimental validation," International Journal of Automation and Computing, vol. 16, no. 3, pp. 310-328, 2019, doi: 10.1007/s11633-018-1165-4.

[13] H. Walid, R. Djamel, M. Sami, "Fractional order direct torque control of permanent magnet synchronous machine," European Journal of Electrical Engineering, vol. 21, no. 5, pp. 431-438, 2019, doi: 10.18280/ejee.210505.

[14] P. Lino, J. Königsmarková, G. Maione, "Feedback-Feedforward Position and Speed Control of DC Motors by Fractional-Order PIv Controllers," 2019 IEEE International Conference on Systems, Man and Cybernetics SMC, Bari, Italy, pp. 2584-2589, 2019, doi: 10.1109/SMC.2019.8914031.

[15] Z. Wu, J. Yuan, Y. Chen, D. Li, Y. Q. Chen, "Fractional order-[PI] controller and Smith like predictor design for a class of high order systems," Arxiv.org/1904.07907, pp. 1-6, 2019.

[16] S. K. Vavilala and T. Vinopraba, "Design of Fractional Order Controllers for Conical Tank Process," 2018 International Conference on Emerging Trends and Innovations In Engineering And Technological Research ICETIETR, Ernakulam, pp. 1-5, 2018, doi: 10.1109/ICETIETR.2018.8529047.

[17] G. Gandikota and D. K. Das, "An FO- $\mathrm{I}^{\lambda} \mathrm{D}^{1-\lambda}$ controller design and realization for inverted decoupled two input two output-liquid level system," International Journal of Dynamics and Control, vol. 8, pp. 1013-1026, 2020, doi: 10.1007/s40435-019-00602-0.

[18] A. Tepljakov, E. Petlenkov, J. Belikov, "FOMCON: A MATLAB toolbox for fractional order system identification and control," International Journal of Microelectronics and Computer Science, vol. 2, no. 2, pp. 51-62, 2012.

[19] B. Vanavil, K. Krishna Chaitanya, A. Seshagiri Rao, "Improved PID controller design for unstable time delay processes based on direct synthesis method and maximum sensitivity," International Journal of Systems Science, vol. 46, no. 8, pp. 1349-1366, 2013, doi: 10.1080/00207721.2013.822124.

[20] C. Ravikishore, D. T. V. Praveen Kumar, R. Padma Sree, "Enhanced performance of PID controllers for unstable time delay systems using direct synthesis method," Indian Chemical Engineer, pp. 1-18, 2020, doi: 10.1080/00194506.2020.1736650.

[21] F. J. Castillo-Garcia, V. Feliu-Batlle, R. Rivas-Perez , "Time domain tuning of fractional order controllers combined with a Smith predictor for automation of water distribution in irrigation main channel pools," Asian Journal of Control, vol. 15, no. 3, pp. 819-833, 2013, doi: 10.1002/asjc.558.

[22] A. Kumar, M. N. Anwar, "Parallel control structure scheme for load frequency controller design using direct synthesis approach," International Journal of Electrical and Computer Engineering, vol. 10, no. 1, pp. 47-60, 2020, doi: 10.11591/ijece.v10i1.pp47-60.

[23] Ch. Anil, R. Padma Sree, "Tuning of PID controllers for integrating systems using direct synthesis method," ISA Transactions, vol. 57, pp. 211-219, 2015, doi: 10.1016/j.isatra.2015.03.002.

[24] M. N. Anwar, S. Pan, "A new PID load frequency controller design method in frequency domain through direct synthesis approach," International Journal of Electrical Power and Energy Systems, vol. 67, pp. 560-569, 2015, doi: 10.1016/j.ijepes.2014.12.024.

[25] M. Kumar, R. S. Singh, "PID controller design for SOPDT using direct synthesis method," International Journal Intelligent Systems and Applications, vol. 9, pp. 56-64, 2019, doi: 10.5815/ijisa.2019.09.06.

[26] H. W. Bode, Network analysis and feedback amplifier design, New York: Van Nostrand, 1945.

[27] A. Kasbi, A. Rahali, "Doubly fed induction generator based variable speed wind conversion system control enhancement by applying fractional order controller," World Academy of Science, Engineering and Technology, International Journal of Energy and Power Engineering, vol. 13, no. 11, pp. 693-709, 2019, doi: 10.5281/zenodo.3566435.

[28] L. L. Ye, J. Q. Bing, "Analytical optimization of IMC-PID design based on performance/robustness trade-off tuning strategy for the modified Smith structure," Journal of Low Frequency Noise, Vibration, and Active Control, vol. 39, no. 1, pp. 158-173, 2019, doi: 10.1177/1461348419829946.

[29] E.Yumuk, M. Güzelkaya, İ. Eksin, "Analytical fractional PID controller design based on Bode's ideal transfer function plus time delay," ISA Transactions, vol. 91, pp. 196-206, 2019, doi: 10.1016/j.isatra.2019.01.034.

[30] B. Keziz, A. Djouambi, S. Ladaci, "A new fractional order controller tuning method based on Bode's ideal transfer function," International Journal of Dynamics and Control, vol. 8, pp. 932-942, 2020, doi: 10.1007/s40435-02000608-z.

[31] M. Safaei, S. Tavakoli, "Analytical fractional PID controller design based on Bode's ideal transfer function plus time delay," International Journal of Dynamical systems, vol. 6, pp. 179-187, 2018, doi: 10.1007/s40435-0170312-z.

IAES Int J Rob \& Autom, Vol. 10, No. 1, March 2021:`10-23 
[32] P. P. Arya, S. Chakrabarthy, "IMC based fractional order controller design for specific non-minimum phase systems," in Proceedings of IFAC Conference on Advances in Proportional-Integral-Derivative Control, vol. 51, no. 4, pp. 847-852, 2018, doi: 10.1016/j.ifacol.2018.06.123.

[33] N. Paliwal, L. Srivastava, M. Pandit, "PSO-based PID controller designing for LFC of single area electrical power network," Nature Inspired Optimization for Electrical Power System, Springer, pp. 43-54, 2020, doi: 10.1007/978981-15-4004-2_4.

[34] M. Morrari and E. Zafiriou, Robust Process Control, Englewood Cliffs, New Jersey: Prentice Hall, 1989.

[35] S. K. Vavilala, T. Vinopraba, "Tuning of the two degree of freedom FOIMC based on the Smith predictor", International Journal of Dynamics and Control, Springer, 2021, https://doi.org/10.1007/s40435-020-00742-8.

\section{BIOGRAPHIES OF AUTHORS}

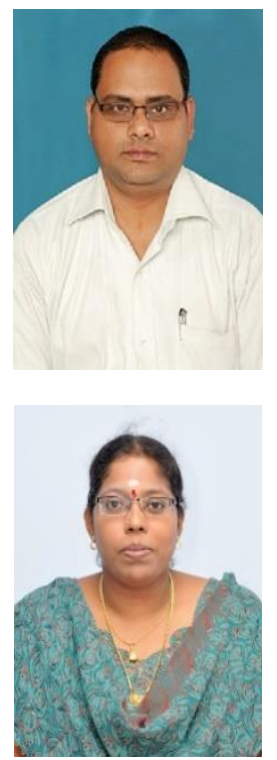

Sateesh K. Vavilala is a research scholar in the Department of Electrical and Electronics Engineering, at NIT Puducherry, Karaikal, India. He completed M. Tech in Instrumentation and Control Systems from Dept. Of Electrical Engineering, NIT Calicut in 2001. His research interests are nonlinear systems, fractional order control, and adaptive control.

Vinopraba Thirumavalavan is working as Assistant professor in the Department of Electrical and Electronics Engineering, at NIT Puducherry, Karaikal, India. She completed Ph.D. from Dept. of Instrumentation and Control, NIT Tiruchirapalli in 2013. Her research interests are fractional order PID controller and Instrumentation system design. 